\title{
Proposta de melhorias em ferramenta de deteção de bordas em imagens termográficas de mamas
}

\section{Proposal for improvements in edge detection tool in thermographic images of breasts}

Lucélio Dias de Azevedo ${ }^{1}$, Simone Vasconcelos Silva ${ }^{2}$, Everton Alves Miranda ${ }^{3}$

\section{RESUMO}

O câncer de mama é uma das principais doenças que atingem as mulheres, como a temperatura do corpo sempre foi utilizada para mensurar o sinal de saúde, a Termografia pode auxiliar na detecção de câncer. A Termografia é uma técnica sem contato físico, não invasiva rápida e pode monitorar uma grande área do corpo. Este trabalho tem como objetivo abordar as técnicas de detecção de bordas em imagens, principalmente em imagens termográficas utilizadas para auxiliar a detecção de anomalias nas mamas, além de sugerir uma redução no custo de aquisição das imagens através do uso de câmeras de aparelhos celulares. Este trabalho apresenta um método específico e uma ferramenta para detecção de bordas em imagens termográficas das mamas, detalha algumas de suas aplicações e propõem melhorias.

Palavras-chave: Termografia, Detecção de Borda, Mama, Imagens, Ferramenta.

\section{ABSTRACT}

Breast cancer is one of the main diseases affecting women, such as Body temperature has always been used to measure the health signal, thermography can assisting in the detection of cancer. Thermography is a technique without physical contact, noninvasive, Fast and can monitor a large area of the body. This work aims to address the techniques of detecting edges in images, mainly in thermographic images. The detection of anomalies in the breasts, in addition to suggesting a reduction in the Cost of acquiring images through the use of mobile camera devices. This has a specific method and a tool for detecting edges in Thermographic images of the breasts, details some of its applications and proposes improvements.

Keywords: Thermography, edge detection, breast, images, tool.
Mestrando em Sistemas Aplicados à Engenharia e Gestão Instituto Federal de Educação, Ciência e Tecnologia Fluminense E-mail: luceliodias@msn.com

2 Doutora em Computação Instituto Federal de Educação, Ciência e Tecnologia Fluminense E-mail: simonevs@iff.edu.br

Mestrando em Sistemas Aplicados à Engenharia e Gestão Instituto Federal de Educação, Ciência e Tecnologia Fluminense E-mail: eamirand@iff.edu.br 


\section{INTRODUÇĀOO}

De acordo com o INCA (2018) pode-se afirmar que o câncer de mama é ainda relativamente incomum antes dos 35 anos, com sua incidência aumentando progressivamente depois dos 50 anos, e no Brasil, para o ano de 2016, foram estimados 57.960 novos casos. Boa parte dos casos de câncer de mama tem bons prognósticos, se o diagnóstico correto for realizado precocemente. Segundo informações de ACS (2018), não existe uma maneira segura de prevenir o câncer de mama, porém ações podem ser realizadas a fim de diminuir o risco de aparecimento do mesmo, como manter seu peso em faixas saudáveis, ser fisicamente ativo e limitar o consumo de álcool. O IBBC (2018) lista os diferentes tipos de exames que podem ser utilizados para detectar o câncer de mama, como: o autoexame, exame clínico, exame de sangue, mamografia, ultrassonografia, ressonância magnética ou biópsia das mamas.

Lahiri et al. (2012) explica que a temperatura do corpo sempre foi utilizada para mensurar o sinal de saúde. A Termografia é uma técnica sem contato físico, nãoinvasiva, rápida e pode monitorar uma grande área do corpo. Pesa também o fato de ser uma tecnologia que pode ser utilizada em tempo real, obtendo as variações dinâmicas de temperatura, bem como não possuir efeitos de radiações prejudiciais. Silva (2010) menciona que o corpo humano emite radiação térmica e uma câmera térmica faz a captura e converte a mesma em uma imagem que representará as diferentes temperaturas do corpo. Neste caso, existem fatores básicos que determinam a emissão de calor do corpo humano: taxa metabólica básica, atividade específica do órgão e a atividade muscular no local onde a temperatura será mensurada.

Este trabalho tem como objetivo geral analisar técnicas de extração de bordas, principalmente extração de borda de imagem termográfica que visa extrair características geométricas da mama de pacientes, abordando a utilização das mesmas. Faz uma análise detalhada de método e ferramenta para extração de bordas em imagens termográficas das mamas, sugerindo formas para redução no custo de aquisição das imagens através do uso de câmeras para aparelhos celulares, além de sugerir uma versão mobile para a ferramenta estudada. 


\section{MATERIAIS E METODOS}

\subsection{Imagens térmicas no diagnóstico de anomalias da mama}

Lanisa et al. (2017) indicam que o método de triagem de termografia chamou a atenção para ser usado como ferramenta alternativa de prevenção devido ao seu procedimento sem contato, além de estar disponível para pacientes com menos de 40 anos. Neste estudo, a imagem da termografia é usada como imagem de entrada dos testes.

Para Thamilselvi et al. (2017) termografia é uma ferramenta adicional para o diagnóstico precoce de câncer de mama. Neste trabalho, uma tentativa é feita para extrair o mapa de borda das imagens de mama com uma segmentação precisa, utilizando um filtro não-linear iterativo que reduz o ruído e preserva as bordas com base na topologia da imagem local.

Araújo et al. (2017) propõem uma abordagem inovadora para classificar as anormalidades da mama (maligna, benigna e cística), empregando dados de intervalo de temperatura para detectar câncer de mama. Os autores comparam os desempenhos da abordagem proposta e de muitos métodos extraídos da literatura de classificação de dados de intervalo para a termografia mamária. Os resultados mostram que o método superou os algoritmos concorrentes.

Lashkari et al. (2016) comparam uma técnica altamente supervisionada com uma técnica não supervisionada que usa imagens térmicas de mama com o objetivo de auxiliar os médicos na detecção precoce de câncer de mama e conclui-se que a técnica sem supervisão pode ser adequada para determinar áreas suspeitas em comparação com uma técnica supervisionada, tendo precisão média de $88 \%$.

Guzman-Cabrera et al. (2016) apresentam uma abordagem de processamento digital que permite a identificação e posterior isolamento da região de interesse para a análise. Os resultados são apresentados com diferentes tipos de câncer em homens e mulheres e variados ângulos de imagem que demonstram a robustez do método proposto.

Tepper et al. (2015) utilizam a termografia para determinar a diferença de temperatura do tumor e a correlaciona com a taxa de crescimento do mesmo. Portanto, uma imagem térmica do tumor pode ser um método de monitoramento muito útil, uma vez que as mudanças térmicas ocorrem antes das mudanças físicas. 
O monitoramento contínuo da diferença de temperatura do tumor produz informações valiosas sobre o estado do tumor e sua agressividade e pode ser realizado em uma única imagem, ou de maneira contínua, em imagens múltiplas que revelam mudanças no estado tumoral.

No trabalho realizado por Heriana et al. (2015) os resultados das imagens capturadas pelas câmeras infravermelhas foram processados em algoritmos de computação para classificar o câncer nas áreas mamárias de acordo com as características de cada imagem. As variações de emissão de radiação infravermelha das imagens capturadas mostraram o nível de câncer.

O trabalho de Silva (2010) aborda a definição de um método para geração de um modelo 3D da mama através da extração de curvas de imagens termográficas de pacientes. Além do método, também foi desenvolvida uma ferramenta computacional (Thermic Breast 3D) a partir de uma metodologia dividida em três partes: (i) Geração do modelo 2D, (ii) Geração do modelo 3D, (iii) Teste e validação do modelo.

\subsection{Técnicas de extração de bordas}

A extração de bordas pode ser entendida como a identificação e localização de pontos de descontinuidade em uma imagem, podendo utilizar diversos algoritmos, tais como:

- $\quad$ Canny Edge Detector: De acordo com Canny (1986), a detecção e extração de bordas serve para simplificar a análise das imagens, reduzindo a quantidade de dados a serem processados, enquanto ao mesmo tempo preserva informações estruturais úteis sobre os limites do objeto. Foi desenvolvido um modelo matemático que pode ser usado para detectar bordas e foram utilizados três na detecção: (i) Boa detecção - deve existir baixa probabilidade de falha ao serem marcados os pontos reais da borda, com funções que diminuem a saída de ruídos na detecção; (ii) Boa localização - os pontos marcados como bordas devem ser o mais próximo possível do centro da real borda; (iii) uma única resposta para uma única borda - implicitamente capturado pelo primeiro critério, quando existirem duas resposta para a mesma borda, uma deve ser considerada falsa;

- $\quad$ Laplacian of Gaussian Filter (LoG): Esta técnica (Huertas, 1986) inicialmente aplica filtro Gaussian à imagem, posteriormente fazendo o Laplacian, verificando os cruzamentos entre os mesmo com a finalidade de destacar as bordas da imagem. $O$ 
primeiro passo borra a imagem com o filtro Gaussian, a fim de tornar o filtro Laplacian (utilizado no segundo passo) menos suscetível aos ruídos da imagem. Estes dois passos compõe o LoG Filter.

- Roberts Cross Edge Detector: Em Zhu e Wu (2017) é demonstrada que esta técnica executa uma medição rápida sobre o gradiente espacial 2D de uma imagem, realçando o que normalmente corresponde à borda da imagem. A base deste algoritmo consiste em dois núcleos de matriz 2x2 trabalhando sobre uma imagem separadamente, a fim de encontrar as bordas gradientes da imagem;

- Sobel Filter: O trabalho descrito em Gao et. al (2010) descreve que o Sobel Filter possui duas grandes vantagens em comparação com outros métodos: (i) introdução do fator médio, que tem efeito na suavização no ruído aleatório da imagem e (ii) melhora nos elementos da borda de ambos os lados, fazendo com que a mesma pareça grossa e brilhante. Zhu e Wu (2017) explica que no Sobel Filter, cada ponto da imagem é envolvido em dois núcleos, onde o primeiro geralmente responde à borda vertical, e o segundo geralmente responde à borda horizontal. $\mathrm{O}$ valor máximo das duas situações é o valor de saída do ponto e o resultado da operação é uma imagem de margem. Sobel operador tem ainda melhor processamento para as imagens com gradientes cinza e muito ruído, porém seu custo computacional é grande;

- $\quad$ Limiarização (Threshold) é uma importante abordagem para segmentação de imagens, onde o processo é uma forma de extrair objetos do fundo de uma imagem através da seleção de um limiar T que separe os dois grupos (objeto e fundo da imagem). Os pixels rotulados como 1 correspondem aos objetos e aqueles rotulados como 0 corresponde ao fundo. Quando $T$ depende apenas de $f(x, y)$, o limiar é chamado de global, mas quando depende de $f(x, y)$ e de alguma propriedade local do ponto $p(x, y)$, então o limia é chamado dinâmico. Na definição do limiar pode-se usar informações do histograma da imagem, o qual associa a cada intensidade de cor na imagem a sua frequência de ocorrência. $E$ para determinar os pixels de uma borda da imagem é possível utilizar o operador gradiente e um limiar (SONKA, HLAVAC e BOYLE, 2008), (GONZALES e WOODS, 2000). 


\subsection{Um Método para Extração de Bordas em Imagens Termográficas (Modelo 2D)}

O trabalho de Silva (2010) descreve um método para a extração de bordas em imagens termográficas das mamas de pacientes utilizando os seguintes passos:

- Determinação da região de interesse: uma avaliação realizada por profissionais radiologistas em uma amostra de 151 imagens termográficas sugeriu que o limite superior da ROI (Region of Interest) seja acima da posição da axila e pouco abaixo da clavícula, pois nessas regiões ainda se encontra tecido mamário. Desta forma encontra-se o limite superior e inferior da região de interesse das imagens frontal e lateral das mamas, conforme mostra a Figura 1a;

- Detecção dos contornos para extração da borda: uma função que retorna os componentes RGB (Red, Green e Blue) da cor do pixel foi utilizada na detecção de cada pixel da imagem termográfica. A detecção da borda baseou-se nos princípios do processo de limiarização (threshold) incluindo algumas alterações, utilizando o false color e a paleta rainhi (permite perceber que o contorno do corpo da paciente é representado por temperaturas, que na imagem termográfica formam um conjunto de pontos que dão origem às curvas do corpo). O processo threshold analisa cada pixel de uma imagem e o substitui por um pixel preto ou branco conforme seja menor ou maior que um valor de intensidade (limiar). A detecção dos contornos para extração de bordas é dividida em duas partes (Figura 1b): (i) detecção do contorno das bordas que separam o corpo da região externa e (ii) detecção do contorno das bordas inferiores das mamas;

- Plotagem das Curvas 2D: nestaparte do processo, os limites da região de interesse são determinados e os pontos armazenados para plotagem. $\mathrm{Na}$ imagem frontal, a parte acima dos pontos representou a borda por uma reta que une os pontos ao limite superior da região de interesse, assim, aregião acima da axila e abaixo da clavícula também pode pertencer à modelagem. Para determinar as curvas (Figura 2) formadas pelos pontos de contorno encontrados, é utilizado o Método 
dos Mínimos Quadrados (MQQ), que utilizaa distribuição de pontos para ajustar a melhor curva ao conjunto de dados.

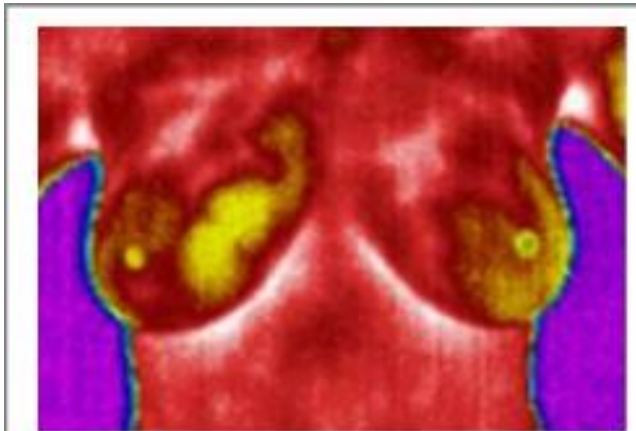

(a)
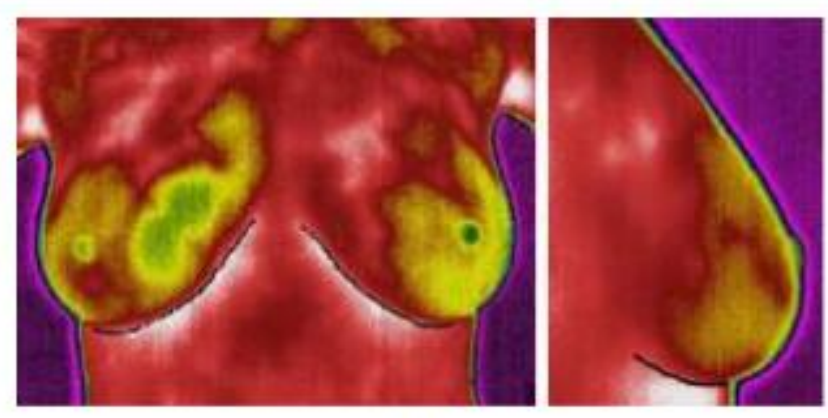

(b)

Figura 1 - (a) ROI da imagem. (b) Contornos (bordas) da mama. Fonte: Silva (2010).

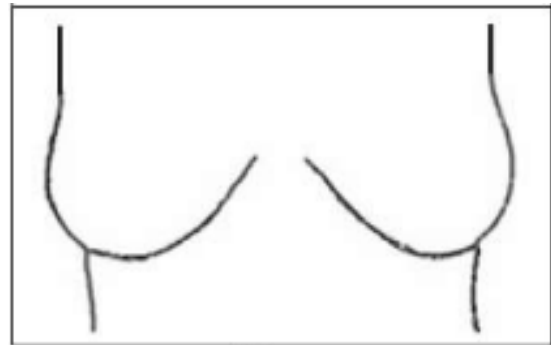

(a)

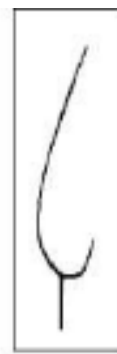

(b)

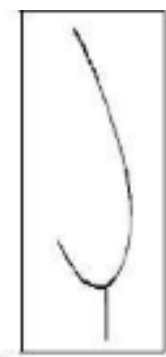

Figura 2 - Plotagem das curvas do Modelo 2D (a) frontal (b) lateral. Fonte: Silva (2010).

\subsection{Aplicações}

Pode - se detalhar duas aplicações importantes que utilizaram o Modelo 2D:

- Ferramenta Thermic Breast 3D: De acordo com Silva (2010), utilizando o Modelo 2D foi possível implementar a ferramenta para geração do Modelo 3D que representa a imagem real do exame termográfico da paciente. $\mathrm{Na}$ ferramenta, após abrir o arquivo da imagem, o usuário seleciona o tipo frontal ou lateral. Assim, a ferramenta marca automaticamente os pontos de contorno que separam o interior do exterior do corpo (lateral do tronco) e a parte inferior da mama da paciente. Através desses pontos, a ferramenta gera um Modelo 2D aproximado das medidas e formas reais da paciente, conforme mostra as Figura 7. Automaticamente é feita a conversão de todos os pontos $(\mathrm{x}, \mathrm{y})$ de pixels para milímetro com o objetivo de proporcionar medições no modelo, tais como, área, volume, e outras, visto que esse 
padrão de medida é um dos mais utilizados pelos softwares que trabalham com malhas. A adequação de escala entre pixels e milímetros é realizada através do seguinte procedimento: (i) Aquisição da medida da paciente -posicionada na vertical entre dois anteparos e amparada por uma superfície plana (normalmente uma das paredes da sala de exame); (ii) Aquisição da medida do Modelo 2D-a ferramenta localiza no Modelo2D frontal o ponto, pertencente à curva do lado esquerdo do tronco, que esteja imediatamente abaixo do ponto mínimo da curva inferior da mama, o mesmo procedimento é feito para curva do lado direito; (iii) Conversão automática pela ferramenta: primeiramente, a medida obtida da paciente em centímetro é convertida para milímetro, e em seguida é feita uma regra de três simples para conversão dos pontos $(x, y)$ em pixels para pontos $(x, y)$ em milímetros. A geração do Modelo 3D (Figura 3) é feita utilizando os pontos extraídos do Modelo 2D, onde através desses pontos foram calculados os pontos de controle para geração das NURBS (Non Uniform Rational BSplines Surfaces) (Azevedo e Conci, 2003), utilizando a biblioteca gráfica OpenGL. A ferramenta permite ao usuário optar pela geração do Modelo 3D contendo as mamas e parte do tronco, bem como somente as mamas, separadamente;

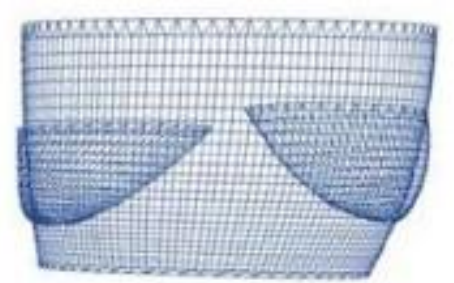

Figura 3 - Malha 3D. Fonte: Silva (2010).

- Geometria Substituta Determinada A Partir De Próteses Mamária: A extração das curvas através do Modelo 2D, foi utilizada para auxiliar uma pesquisa de aperfeiçoamento na escolha de próteses mamárias externas para pacientes, selecionando os modelos das próteses que mais se aproximavam das medidas e curvas reais de pacientes. Esta aplicação foi demonstrada por Viana et. al. (2010), onde usou as geometrias substitutas obtidas através de próteses mamárias externas (Figura 4a) iguais às que são doadas pelo Sistema Único de Saúde (SUS) às pacientes 
mastectomizadas. Para comparação com as próteses, as curvas da base e do perfil da mama da paciente foram retiradas dos termogramas através do Modelo 2D da ferramenta Thermic Breast 3D. Para a obtenção das coordenadas dos pontos que formam cada um dos perfis das próteses foi utilizada uma ferramenta de aplicação para engenharia reversa denominada scan-pack, sendo os pontos obtidos com a máquina funcionando no modo automatizado via comando numérico computadorizado. Para selecionar a geometria substituta mais adequada à paciente foi feita uma comparação entre a curva da base da prótese extraída pelo scan-pack e a curva da base da mama extraída pelo Thermic Breast 3D, desta forma a geometria escolhida é aquela que apresenta o menor erro entre os coeficientes das duas curvas (Figura 4b).

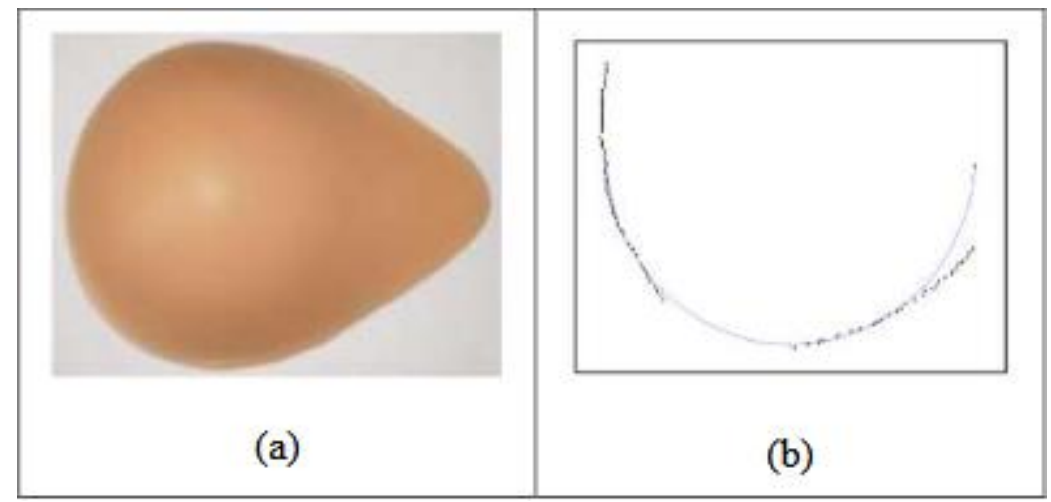

Figura 4 - (a) Prótese mamária externa. (b) Superposição da curva extraída da termografia com a curva extraída da prótese. Fonte: Viana et. al (2010).

\section{RESULTADOS}

Como a proposta do trabalho está em processo de desenvolvimento, pode-se apenas trabalhar com resultados preliminares. Uma vez que o estágio de desenvolvimento do aplicativo está avançado, o port do código para o sistema operacional Android mostra-se viável, como indica a Figura 5. É importante ressaltar que mais testes de compatibilidade com diferentes imagens devem ser realizados, porém, até o momento, o estudo se mostra bastante promissor no que se diz à detecção de bordas e plotagem dos pontos $\mathrm{x}$ e y encontrados. 

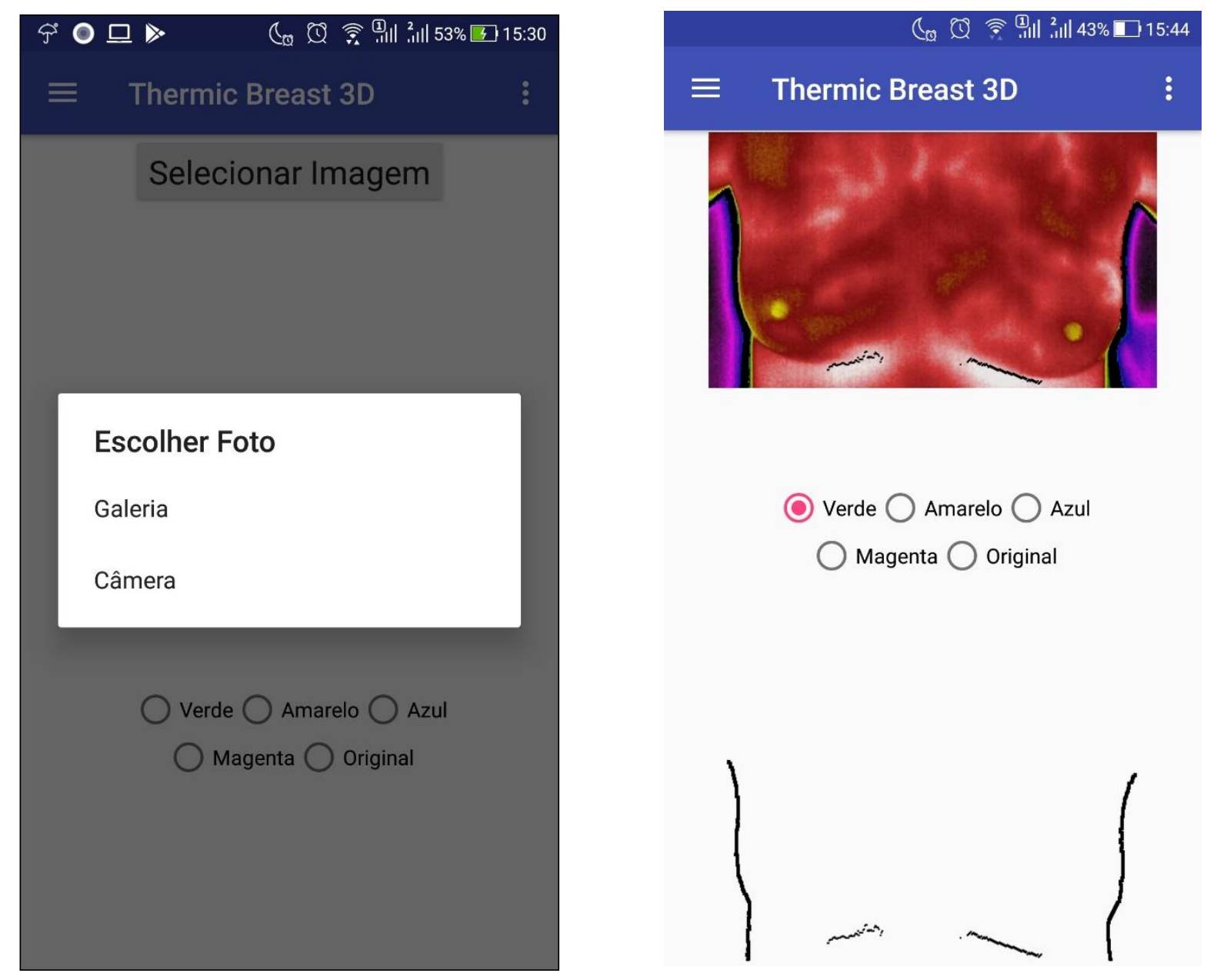

Figura 5 - Imagens do Thermic Breast 3D, versão mobile. Fonte: Os autores.

\section{DISCUSSÁO}

Com base nas informações dos modelos de câmeras térmicas (Tabela 1), pode-se observar que as câmeras térmicas mais utilizadas atualmente (FLIR S45 e FLIR T650sc) possuem alta resolução, mas apresentam um custo muito elevado (cerca de $R \$$ 200.000,00), baixa mobilidade devido a uma série de protocolos existentes para seu transporte e manuseio, além de elevado custo de calibração e manutenção. Já as câmeras térmicas com interação com Smatphones (FLIR One Pro e Cat S61), apesar de possuírem uma resolução inferior, possuem um custo significantemente mais baixo e total mobilidade.

Observando a Tabela 1 o modelo FLIR One Pro apresenta o melhor custo/benefício já que o mesmo pode ser acoplar aos aparelhos de Smartphone já disponíveis no mercado. 
Tabela 1 - Características de Modelos de Câmeras Termográficas

\begin{tabular}{|c|c|c|c|c|}
\hline $\begin{array}{c}\text { Modelo de } \\
\text { Câmera }\end{array}$ & $\begin{array}{c}\text { Resolução } \\
\text { Térmica }\end{array}$ & Temperatura & Preço & Tipo \\
\hline FLIR S45 & $320 \times 240$ pixels & $-40^{\circ} \mathrm{C}$ a $2.000^{\circ} \mathrm{C}$ & $\mathrm{R} \$ 189.000,00$ & Câmera Térmica \\
\hline FLIR T650sc & $640 \times 480$ pixels & $-40^{\circ} \mathrm{C}$ a $2.000^{\circ} \mathrm{C}$ & $\mathrm{R} \$ 200.000,00$ & Câmera Térmica \\
\hline $\begin{array}{c}\text { FLIR One } \\
\text { Pro }\end{array}$ & $160 \times 120$ pixels & $-20^{\circ} \mathrm{C}$ a $400^{\circ} \mathrm{C}$ & $\mathrm{R} \$ 1.600,00$ & $\begin{array}{c}\text { FLIR acoplada ao } \\
\text { Smartphone }\end{array}$ \\
\hline Cat S61 & $160 \times 120$ pixels & $-20^{\circ} \mathrm{C}$ a $400^{\circ} \mathrm{C}$ & $\mathrm{R} \$ 4.300,00$ & $\begin{array}{c}\text { Smartphone com } \\
\text { câmera térmica } \\
\text { FLIR integrada }\end{array}$ \\
\hline
\end{tabular}

Como proposta de melhoria na Ferramenta Thermic Breast 3D, este trabalho sugere o desenvolvimento de uma nova versão mobile da ferramenta (Thermic Breast 3D for Android) de forma que a mesma possa ser executada em aparelhos Smartphone acoplados a uma FLIR One Pro.

As imagens utilizadas no Thermic Breast 3D foram adquiridas através da câmera FLIR S45, que gera imagens com resolução de 320x240 pixels. Logo, um dos desafios da proposta "Thermic Breast 3D Mobile" é trabalhar com imagens com metade da resolução utilizada no "Thermic Breast 3D", o que pode afetar diretamente a geração da malha 3D, por exemplo.

Portanto a versão mobile contará inicialmente apenas com o a geração do Modelo 2D, o qual será testado e comparado com os resultados obtidos na versão original. Caso os resultados sejam positivos, novos esforços poderão ser direcionados para adaptar a geração do Modelo 3D na versão mobile. É primordial que o aplicativo "Thermic Breast 3D Mobile" tenha o mesmo comportamento que a ferramenta criada para computadores no que se diz respeito aos resultados gerados. A integração com a API da câmera facilitará a utilização do aplicativo, pois o usuário do mesmo não precisará ficar alternando entre diferentes aplicativos para visualização dos resultados.

\section{CONSIDERACCOES FINAIS}

Devido à multiplicidade de técnicas de detecção de borda disponíveis, este estudo realizou uma breve revisão das mais comumente usadas, abordou uma técnica de detecção em imagens termográficas e demonstrou sua utilização. 
Fornecer uma alternativa não invasiva e não radioativa com a comodidade de fácil utilizaçãosão fatores positivos para a utilização de imagens termográficas no auxílio na prevenção do câncer de mama. A redução dos custos de aquisição e baixa manutenção propõem uma realidade financeira viável e acessível.

Contando ainda que o Android é o sistema operacional mais utilizado no mundo, com

39,98\% de quota de mercado (STATCOUNTER, 2018), uma grande parcela de usuários terá acesso imediato à proposta apresentada, e por último, pode ser muito útil para ajudar na redução das filas de mamografia / tomografia dos hospitais públicos, no caso de exames iniciais e de acompanhamento do tratamento, onde dados recentes mostram que mais de 8,5 milhões de mulheres deixaram de fazer o exame nos últimos anos, seja por desconhecimento ou por não ter acesso a nenhum tipo de tecnologia de prevenção.

É importante ressaltar que a termografia não substitui a mamografia no que diz respeito a diagnóstico conclusivo.

\section{REFERÉNCIAS}

ACS (2018). American Cancer Society - Disponível em: <https://www.cancer.org/cancer /breast-cancer/risk-andprevention/can-i-lower-my-ri.k.html>.Acesso em 15 jan. 2018.

ARAÚJO, M. C; SOUZA, R. M. C. R; LIMA, R. C. F; FILHO, T. M. S. (2017) An interval prototype classifier based on a parameterized distance applied to breast thermographic images. Medical \& Biological Engineering \& Computing. P. 873-884.

AZEVEDO E.; CONCl A. (2003) Computação Gráfica - Teoria e Prática. Vol. I, 1ํㅜㄴ Edição. Rio de Janeiro: Ed. Campus-Elsevier, 353 p.

CANNY, J. A (1986) Computational Approach to Edge Detection. IEEE Transactions On Pattern Analysis And Machine Intelligence, Vol. PAMI-8, № 6.

GAO, W; ZHANG, X; YANG, L; LIU, H. (2010) An improved Sobel edge detection. 3rd International Conference on Computer Science and Information Technology.

GONZALEZ, R.C.; WOODS, R. E. (2000) Processamento de Imagens Digitais. 1ํㅡㄴ Edição. São Paulo: Ed. Edgard Blücher, 509 p

GUZMAN-CABRERA, R., GUZMAN-SEPULVEDA, J.R.; PARADA, A.G.; CISNEROS, M.T.; BALEANU, D. (2016). Digital processing of thermographic images for medical applications. Revista de Chimie 67, p. 53-56.

HERIANA, O; SOESANDI, I. (2015) Tumor size classification of breast thermal image using fuzzy C-Means algorithm. International Conference on Radar, Antenna, Microwave, Electronics, and Telecommunications. 
HUERTAS, A; Medioni, G. (1986) Detection of Intensity Changes with Subpixel Accuracy Using LaplacianGaussian Masks. IEEE Transactions On Pattern Analysis And Machine Intelligence, Vol. PAMI-8, № 5.

INCA. (2018) INCA - CANCÊR - Tipos de Câncer. Disponível em: $<$ http://www2.inca.gov.br/wps/wcm/connect/tiposdecancer/site/home/mama>. Acesso em 15 jan. 2018.

IBBC. (2018) Instituto Brasileiro de Controle do Câncer. Disponível em <http://www.ibcc.org.br/examesdiagnosticos/>. Acesso em 16. Jan. 2018.

LAHIRI, B. B; S. BAGAVATHIAPPAN, T; JAYAKUMAR, J. P. (2012) Medical applications of infrared thermography: A review. Infrared Physics \& Technology 55. p. 221-235.

LANISA, N; CHAI, H. Y.; CHEOK, S.; LIEW, Y, M.; SALIM, M. I. M.; LAI, K. W. (2017) Texture Similarity Analysis of Breast Abnormalities in Infrared Thermal Image. Journal of Medical Imaging and Health Informatics, Volume 7, Number 8, p. 1830-1836.

LASHKARI, A.E.; FIROUZMAND, M. (2016) Early breast cancer detection in thermogram images using AdaBoost classifier and fuzzy C-Means clustering algorithm. 7. p. 113-124.

SILVA, S. V. (2010) Reconstrução da Geometria da Mama a partir de Imagens Termográficas. Niterói: UFF, 134p. Tese (Doutorado em Computação). Instituto de Computação, Universidade Federal Fluminense.

SONKA, M.; HLAVAC, V.; BOYLE, R. (2008) Image Processing, Analysis and Machine Vision. 3th Edition, Thomson.

STATCOUNTER. (2018) Operating System Market Share Worldwide. Disponível em <http://gs.statcounter.com/os-market-share>. Acesso em 09 abr. 2018.

TEPPER, M. G. I. (2015) Monitoring tumor state from thermal images in animal and human models. Medical Physics. p. 1297-306.

THAMILSELVI, J.; KAVITHA, G.; SUJATHA, C. M. (2017) Geometric nonlinear diffussion filter based edgemap extraction and its validation of infrared breast images. Third International Conference on Biosignals, Images and Instrumentation (ICBSII).

VIANA, M. A. V., SILVA, S. V., SANTOS, F. G. C., CONCI, A., ROLIM, T. L., LIMA, R. C. F. (2010) Obtenção de perfis de temperaturas em mamas através da geometria substituta determinada a partir de próteses mamárias externas. VI Congresso Nacional de Engenharia Mecânica (VI CNEM).

ZHU, K; Wu, J. (2017) An Algorithm for Extracting Spray Trajectory Based on Laser Vision. 17th IEEE International Conference on Communication Technology. 